\title{
Apoyo social como moderador del estrés en la salud de los desempleados
}

\author{
Social support as moderator of the stress \\ on unemployed people's health
}

Recibido: junio 20 de 2009 | Revisado: diciembre 12 de 2009 Aceptado: febrero 14 de 2010

\author{
LETICIA GUARINO* \\ Universidad Simón Bolívar, Caracas, Venezuela \\ Víctor SOJO** \\ Universidad Central de Venezuela, Caracas, Venezuela
}

\section{RESUMEN}

El objetivo de la presente investigación fue evaluar el rol moderador de dos indicadores del apoyo social, en la relación entre la duración del desempleo y la salud física y mental de desempleados venezolanos. Para ello, se administraron instrumentos de autorreporte a una muestra de 328 desempleados residentes en la ciudad de Caracas, Venezuela, con el fin de medir las variables de estudio. Los resultados indicaron que el apoyo social percibido actuó como moderador para predecir una mejor salud general y menor presencia de somatización en los desempleados, de tal manera que amortiguó los efectos de una mayor duración del desempleo sobre estos indicadores de salud. Por su parte, el miedo a la revelación no mostró el efecto de moderación que se esperaba, pero reveló efectos directos sobre la mayoría de los indicadores de salud, actuando como una variable de riesgo para los desempleados. Estos resultados apoyan hallazgos previos sobre la importancia del apoyo social percibido como factor amortiguador ante las experiencias de estrés en diversas poblaciones, en especial la de los desempleados. Asimismo, registran el impacto negativo que tiene la inhabilidad de los individuos para revelar y expresar sus emociones y malestar, cuando están sometidos a la experiencia del desempleo.

Palabras clave autores

Desempleo, duración del desempleo, apoyo social, salud.

Palabras clave descriptores

Psicología social, autorreporte, expresión emocional, investigación cuantitativa.

SICI: 1657-9267(201112)10:3<867:ASMDES>2.3.TX;2-B

Para citar este artículo. Guarino, L. \& Sojo, V. (2011). Apoyo social como moderador del estrés en la salud de los desempleados. Universitas Psychologica, 10 (3), 867-879.

* Dpto. de Ciencia y Tecnología del Comportamiento. Edificio de Estudios Generales, 1er. Piso, Valle de Sartenejas, Aptdo. Postal 89.000-A. Miranda, Venezuela.Tel.:00582129063595.E-mail: lguarino@ usb.ve

** Escuela de Psicología. Edificio Facultad de Humanidades y Educación. Ciudad Universitaria de Caracas. Los Chaguaramos. Caracas, Venezuela. Código postal: 1041. Tel.: +61-8344-6345, Móvil: +61-4387-06447.E-mails: victor.sojo@ucv.ve; vsojo@pgrad.unimelb.edu.au

\section{A B S T R A C T}

The aim of this research was to assess moderating role of two indexes of social support in the relationship between the length of unemployment and the physical and mental health of Venezuelan unemployed. Self-report measures were administered to a sample of 328 unemployed residents in Caracas, Venezuela. Results indicated that perceived social support acted as moderator in predicting better general health and lower somatization in the unemployed, acting as buffer of longer periods of unemployment over these health indexes. On the other side, the fear of disclosure did not show moderator effects as expected, but showed direct effects over the majority health indexes, acting as a risk factor for the unemployed. These results support previous findings regarding the significance of perceived social support as a buffer factor in front of stressful experiences in different populations. At the same time, results showed the negative impact of the individual's inability to reveal and express his/her emotions and distress, when under the experience of unemployment.

Key words authors

Unemployment, length of unemployment, social support, health.

Key words plus

Social psychology, self-report, emotional expression, quantitative research. 


\section{Introducción}

El desempleo es un evento estresante que puede afectar el bienestar psicológico y físico de las personas (Dooley, Fielding \& Lennart, 1996), dependiendo de los recursos de los que dispongan para afrontarlo y de otras variables que facilitan o inhiben su efecto. En esta investigación se pretende determinar si el apoyo social funciona como moderador de la relación entre el estrés asociado al desempleo -específicamente la duración del desempleo- y la salud, en un grupo de desempleados venezolanos.

Un mayor tiempo de desempleo parece impactar negativamente el equilibrio psicosocial y las funciones cognitivas del individuo. En este sentido, Álvaro (1992) encontró que las personas con más tiempo desempleadas reportan necesitar más tiempo para hacer las mismas cosas, concentrarse y llevarlas a cabo con la misma habilidad que antes. Asimismo, estos desempleados expresan tener más dificultades en comenzar alguna tarea, mantenerse mentalmente activos, recordar cosas, tomar decisiones y comprender con rapidez lo que otras personas dicen. Sin embargo, Artazcoz, Benach, Borrell y Cortès (2004) no encontraron tal asociación significativa entre el tiempo desempleado y la salud mental, generándose una inconsistencia con respecto a esta relación.

Varios investigadores han señalado que tales diferencias e inconsistencias observadas entre los resultados, se pueden deber al efecto moderador de algunas características personales y factores psicosociales, en la relación entre eventos estresores (en este caso la duración del desempleo) e indicadores de salud, que pueden resultar protectores o de riesgo, dependiendo de la circunstancia (p. ej., Cohen \& Edwards, 1989; Guarino, 2004; Sandín, 1995; Steptoe, 1991; Taylor \& Aspinwall, 1996). En esta investigación, específicamente, se intenta determinar si dos indicadores del apoyo social, uno disposicional (visto como diferencia individual), conjuntamente con el apoyo social percibido, actúan como moderadoras de la salud de los desempleados en función del tiempo, sometidos a esta condición.
El apoyo social disposicional se refiere a la tendencia o disposición de los individuos para recurrir a otros o no hacerlo en búsqueda de apoyo, constituyéndose en una dimensión más subjetiva e individual del apoyo social. Para evaluarlo, Forbes y Roger (1999) diseñaron el Interpersonal Trust Questionnaire (ITQ, por sus siglas en inglés), con el cual miden las dimensiones de Miedo a la Revelación (MR), Afrontamiento Social (AS) e Intimidad Social (IS). Una adaptación del instrumento a población hispanoparlante (Guarino \& Sojo, 2009) replicó solo los factores de MR y AS, observándose que mientras el miedo a la revelación se asocia negativamente con indicadores de inteligencia emocional y afrontamiento racional, a la vez se relaciona positivamente con indicadores de distrés, sensibilidad emocional negativa, afrontamiento emocional y de evitación. Por su parte, la dimensión de afrontamiento social correlaciona en la dirección inversa con tales indicadores. Si bien no se han encontrado estudios específicos que relacionen el apoyo social disposicional con el estrés del desempleado, se podría esperar que las dimensiones del ITQ, como medidas de la capacidad para buscar apoyo social, actúen en forma diferencial, a la hora de moderar los efectos del estrés, sobre la salud de individuos bajo esta condición.

La literatura con respecto al efecto amortiguador del apoyo social percibido en el estrés de los desempleados, es también muy escasa. En este sentido, solo se tienen las referencias de los estudios de Gore (1978), Gascón, Olmedo, Bermúdez, García-Campayo y Ciccotelli (2003) y Howe, Lockshin y Caplan (2004). Tal vez sea el estudio de Gore (1978) el pionero en el establecimiento del efecto amortiguador del apoyo sobre la salud de los desempleados, al hallar que los individuos en esta condición con bajo apoyo mostraron significativamente más cambios y elevaciones en sus niveles de colesterol, síntomas de enfermedad y respuestas afectivas, comparativamente con los desempleados con fuerte apoyo social.

Por su parte, Howe et al. (2004) evaluaron tres modelos para dilucidar los vínculos entre las relaciones sociales, el estrés producto del desempleo y la salud de las personas, en 252 parejas. En ese 
trabajo se encontraron evidencias de que los estresores comunes generaban síntomas depresivos en ambos miembros de la pareja, así como la rabia y los síntomas depresivos de cada miembro mediaron parcialmente estos efectos en el otro, tal como ocurrió con la calidad de vida. Esto último implica que el tipo de relación que mantienen las personas casadas y el apoyo que se brindan mutuamente, pueden servir como un factor amortiguador si éstos son saludables, aumentando el riesgo de padecer los síntomas descritos en ambos miembros de la pareja en el caso de relaciones "no saludables" o de apoyo. Resultados similares sobre los beneficios del apoyo social obtenido a través de la pareja y de un "buen matrimonio" habían ya sido señalados a partir de un estudio epidemiológico (Sandín, 1995).

Por último, Gascón et al. (2003) estudiaron variables como estrés, calidad de vida, estrategias de afrontamiento, estilos conductuales y síntomas somáticos, en un grupo de desempleados españoles, revisando las interacciones entre estas y comparando sus valores con los reportados por personas empleadas. Los resultados indicaron que el grupo de desempleados manifestó significativamente más estrés diario, peor calidad de vida y más quejas somáticas que el grupo de trabajadores. Con respecto a la relación apoyo social-síntomas somáticos en los desempleados, las correlaciones halladas entre estas dos variables fueron todas negativas y significativas, sugiriendo el papel protector que el apoyo social puede tener sobre el estatus de salud de los desempleados, a pesar de no haberse estudiado según un modelo de moderación.

En general, son múltiples las evidencias sobre el rol moderador del apoyo social, tanto objetivo como percibido, en la relación estrés-salud en diversas poblaciones. Sin embargo, esta relación no ha sido específicamente explorada en grupos de desempleados, mucho menos se han establecido diferencias con respecto al posible efecto moderador del apoyo social como capacidad de buscar y usar el apoyo apropiadamente y el apoyo percibido en personas sometidas a la experiencia de desempleo, lo cual es el foco de atención del presente estudio.

\section{Método}

\section{Participantes}

328 personas desempleadas residentes en el Distrito Capital de Venezuela, distribuidos en $50.6 \%$ hombres $(n=166)$ y $49.4 \%$ mujeres $(n=162)$; con edad media de 31 años (rango de edad entre 18 y 65 años; $D T=9.43$ ) y que llevaban desempleados 1 año en promedio (rango entre 1 mes y 11 años; $D T=1.57$ años).

\section{Instrumentos}

\section{Variables demográficas}

Fueron evaluadas mediante preguntas directas en el cuestionario, luego de la carta de consentimiento informado y antes del inicio de las otras tres escalas. Se preguntó por la edad, sexo y tiempo en meses como desempleados.

\section{Interpersonal Trust Questionnaire (ITQ)}

Versión hispana de Guarino y Sojo (2009): esta escala de autorreporte tipo Likert está compuesta por 46 reactivos que describen sentimientos, creencias y conductas propias y referidas a otras personas con respecto a la búsqueda y uso del apoyo social ante una situación estresante. Mide dos dimensiones del apoyo social disposicional: 1) Miedo a la Revelación (MR), referido a la inhabilidad o dificultad para confiar en los otros, particularmente cuando se trata de asuntos emocionales y 2) Afrontamiento Social (AS), que evalúa la necesidad de buscar a otros para recibir información o ser escuchado cuando se hace frente a experiencias demandantes. Las personas deben indicar su nivel de acuerdo en un rango que va desde 1 (totalmente en desacuerdo) hasta 4 (totalmente de acuerdo). Los índices de consistencia interna obtenidos con la presente muestra de desempleados para ambas subescalas fueron: $\alpha$ $=0.92$ para MR y $\alpha=0.84$ para AS. 


\section{Escala de Apoyo Social Percibido}

Escala de autorreporte de Ullah, Banks y Warr (1985) con versión en español de Rincón (2003), compuesta por 5 ítems ante los cuales las personas deben señalar la frecuencia con la que se sienten apoyados por otros, en términos de recibir ayuda económica, comentar los problemas diarios, sugerir cosas interesantes que hacer, dar ánimo cuando se sienten decaídos y recibir información acerca de trabajos disponibles. La escala podía ser respondida en un rango de 1 (casi nunca) a 4 (casi siempre). El índice de consistencia interna de la escala para este estudio fue de $\alpha=0.67$.

\section{Cuestionario de Salud General (General Health Questionnaire [GHQ])}

Esta escala, con versión hispana de Molina y Andrade (2002), está compuesta por 28 reactivos que describen síntomas de ansiedad, depresión, somatización y disfunción social, respecto a los cuales los participantes deben reportar la frecuencia relativa con la que los han padecido en las últimas semanas, con un rango de posibilidades desde 4 (No, en lo absoluto), hasta 1 (Mucho más que lo habitual). Las puntuaciones más altas reflejan una mejor salud. Los índices de consistencia interna resultaron óptimos para todas las dimensiones del GHQ en la muestra de estudio, oscilando entre $\alpha=0.80$ y $\alpha$ $=0.93$, con $\alpha=0.93$ en la escala completa.

\section{Procedimiento}

Se desarrolló un estudio de tipo correlacional y transversal por cuestionarios, contactando a los participantes en dos Agencias de Empleo del Ministerio del Trabajo y una Organización No Gubernamental sin fines de lucro; ambas instituciones situadas en Caracas, Venezuela y destinadas al asesoramiento, el entrenamiento y la ubicación laboral. Se solicitó a los desempleados que asistieron a estas agencias a responder los cuestionarios, explicándoles la naturaleza del estudio y precisando que su participación era completamente voluntaria y no vinculada con los servicios de las instituciones donde se les contactó. Los datos fueron sometidos a análisis multivariable, de carácter estrictamente cuantitativo, usando los métodos de regresión jerárquica para comprobar el efecto de moderación del apoyo social sobre los indicadores de salud.

\section{Resultados}

Una breve revisión de los factores sociodemográficos indicó que en esta muestra no se encontraron diferencias entre hombres y mujeres en cuanto a la edad, pero sí referida a la duración del periodo de desempleo, $t(303)=2.09 p<0.05$. Más específicamente, los hombres presentaron en promedio 9 meses y medio como desempleados y las mujeres 1 año y 2 meses. La edad y la duración del desempleo presentan una correlación $r(303)=0.250$ $p<0.001$, lo cual revela que en esta muestra los participantes de mayor edad tienen mucho más tiempo sin trabajo que los jóvenes.

Las respuestas de los participantes a las dimensiones de cada instrumento aplicado fueron promediadas para el cálculo de estadísticos descriptivos y bivariados, para que las puntuaciones reflejaran la escala de respuesta que emplearon los mismos al reportar su apoyo social (disposicional y percibido) y su estado de salud. Tal como se observa en la Tabla 1, las medias de los participantes en las dimensiones de apoyo social (rango de 1 a 4) indican que el afrontamiento social es considerablemente alto, mientras que el miedo a la revelación es moderado, así como el apoyo social percibido puede considerarse moderadamente alto, lo cual se constituye en una condición favorable para esta muestra. En cuanto a los indicadores de salud, el hecho de que todas las medias estén por encima de la puntuación de 3 en este instrumento, indica que los participantes se han sentido bastante mejor física, emocional y socialmente, que de costumbre, a pesar de su situación de desempleo; sin embargo, la puntuación más baja se encuentra en el funcionamiento social.

Al comparar los tipos de apoyo social dependiendo del sexo, se observa que en el apoyo social percibido, las mujeres tienen una puntuación $($ media $=2.94)$ significativamente mayor a la de 
los hombres (media $=2.78)$. Sin embargo, con respecto a los indicadores de salud, las mujeres reportaron significativamente más sintomatología somática, de ansiedad y disfunción social que los hombres, aunque en todos los casos ambos grupos poseen medias superiores a los 3 puntos.

Por otro lado, tanto la edad como la duración del periodo de desempleo se encontraron correlacionadas negativamente con la disfunción social y salud general, aunque en ambos casos las correlaciones son muy bajas. Llama también la atención, el resultado con respecto al apoyo social percibido, siendo los participantes más jóvenes los que perciben más apoyo, a diferencia de los de mayor edad. Lo contrario se observa con relación a la duración del desempleo, ya que mientras más prolongado sea este, mayor inhibición por parte de los individuos en revelar sus sentimientos y emociones, tal como se corrobora en la Tabla 1.

\section{TABLA 1}

Pruebas $t$ para diferencias de sexo y correlaciones bivariadas de edad y Duración del Desempleo con las variables estudiadas

\begin{tabular}{|c|c|c|c|c|c|}
\hline & Media & DT & $t$ sexo & $r_{\mathrm{xy}} \mathrm{Edad}$ & $r_{\mathrm{xy}} \mathrm{DD}$ \\
\hline $\begin{array}{l}\text { Afronta- } \\
\text { miento } \\
\text { Social }\end{array}$ & 3.20 & 0.42 & 0.950 & -0.101 & -0.023 \\
\hline $\begin{array}{l}\text { Miedo a la } \\
\text { Revelación }\end{array}$ & 2.22 & 0.53 & -0.301 & -0.022 & $0.132 *$ \\
\hline $\begin{array}{l}\text { Apoyo } \\
\text { Social } \\
\text { Percibido }\end{array}$ & 2.86 & 0.66 & $2.222 *$ & $-0.171 * *$ & -0.073 \\
\hline $\begin{array}{l}\text { Somatiza- } \\
\text { ción }\end{array}$ & 3.27 & 0.63 & $2.439^{*}$ & -0.065 & -0.071 \\
\hline Ansiedad & 3.31 & 0.69 & $2.065^{*}$ & -0.106 & -0.056 \\
\hline Disf. Social & 3.25 & 0.49 & $2.132 *$ & $-0.188 * *$ & $-0.156^{* *}$ \\
\hline Depresión & 3.77 & 0.49 & 0.353 & -0.028 & -0.059 \\
\hline $\begin{array}{l}\text { Salud } \\
\text { General }\end{array}$ & 3.40 & 0.46 & 1.847 & $-0.158 * *$ & $-0.155^{* * *}$ \\
\hline
\end{tabular}

$\mathrm{DD}=$ Duración del Desempleo

$* * p<0.01 ; * p<0.05$

Fuente: elaboración propia.
Considerando los resultados referidos al sexo y la edad como variables asociadas al apoyo social y a la salud, se decidió controlarlas en los análisis de regresión jerárquica, para evaluar el efecto moderador del apoyo social (disposicional y percibido) en la relación entre la duración del desempleo y la presencia de síntomas, en las cuatro dimensiones del GHQ.

Siguiendo los lineamientos de Aiken y West (1991) y de Baron y Kenny (1986) para el cálculo de interacciones entre variables empleando regresión múltiple para evaluar efectos de moderación, se procedió a la transformación de los valores obtenidos por los participantes en todas las escalas empleadas, en edad y duración del periodo de desempleo a puntajes estandarizados $z$, y el sexo en una variable dummy, creando posteriormente los términos de interacción entre las tres dimensiones de apoyo social y duración del periodo de desempleo.

De esta manera, en un primer paso de las regresiones para predecir cada uno de los indicadores de salud del GHQ, se incluyó el sexo y la edad de los participantes; en un segundo paso, la duración del periodo de desempleo; en el tercer paso, los tres factores de apoyo social (afrontamiento social, miedo a la revelación y apoyo social percibido), y en un último paso, los términos de interacción de estos tres factores con la duración del periodo de desempleo.

La Tabla 2 muestra el modelo resumido para la predicción de la salud general. En el análisis por pasos se observa que la edad hace una contribución significativa, específicamente las personas de mayor edad reportan más deterioro en su salud general. Igualmente, la duración del periodo de desempleo está asociada negativamente con la salud. El miedo a la revelación presenta una asociación inversa y significativa con la salud general, sugiriendo, en este caso, que a mayor dificultad para expresar y revelar las propias emociones y sentimientos en circunstancias estresantes, mayor el deterioro general en los desempleados. En el paso 4 de la ecuación se obtuvo una interacción significativa para la predicción del puntaje de salud general, en este caso entre el apoyo social percibido 
y la duración del desempleo, indicando que esta forma de apoyo social modera la relación entre el estrés del desempleo y la salud general.

\section{TABLA 2}

Modelo de regresión jerárquica para la predicción de la Salud General

\begin{tabular}{lllll}
\hline Modelo & $\begin{array}{c}\text { Coefi- } \\
\text { ciente no } \\
\text { Estanda- } \\
\text { rizado }\end{array}$ & $\begin{array}{c}R^{2} \\
\text { corregida }\end{array}$ & $\begin{array}{c}\text { Cambio Cambio } \\
\text { en } R^{2}\end{array}$ & en F \\
\hline 1 Paso 1 & & 0.018 & 0.028 & $4.172^{*}$ \\
Sexo & 0.116 & & & \\
Edad & $-0.156^{* *}$ & & & \\
2 Paso 2 & & 0.028 & 0.014 & $4.181^{*}$ \\
$\begin{array}{l}\text { Duración del } \\
\text { desempleo }\end{array}$ & $-0.132^{*}$ & & & \\
3 Paso 3 & & 0.105 & 0.085 & $9.289^{* *}$ \\
Afrontamien- & 0.085 & & & \\
to Social & & & & \\
Miedo a la & $-0.245^{* *}$ & & & \\
Revelación & & & & \\
Apoyo Social & 0.069 & & & \\
Percibido & & & &
\end{tabular}

4 Paso 4

$0.147 \quad 0.049 \quad 5.669 * *$

Afrontamien to Social x DD ${ }^{-0.074}$

Miedo a la Revelación x DD -0.035

Apoyo Social

Percibido $x \quad 0.248^{* *}$

DD

$\mathrm{DD}=$ Duración del Desempleo

$* * p<0.01 ; * p<0.05$

Fuente: elaboración propia.

En las pendientes simples para la interacción entre el apoyo social percibido y la duración del periodo de desempleo en su efecto sobre la salud general (Preacher, Curran \& Bauer, 2006), se observó que las personas que perciben un mayor apoyo social en su contexto (más 1 DT de la media) presentan un mejor estado de salud que los demás participantes, aunque esta diferencia no es significativa $(b=0.14, t=1.68, p=0.09)$. Sin embargo, en el caso de los individuos con un bajo apoyo social percibido (menos 1 DT de la media), un mayor tiempo de desempleo genera un mayor deterioro en su salud general $(b=-0.35, t=-3.11, p=0.002)$. Esta relación está ilustrada en la Figura 1.

En la Tabla 3 se puede observar que de todas las variables estudiadas en la predicción de la depresión, solo el afrontamiento social y el miedo a la revelación se relacionan significativamente con este indicador de salud, aunque en formas contrarias. Así, mientras mayor sea el uso de recursos sociales y la búsqueda de apoyo en situaciones estresantes (AS), menor la probabilidad de desarrollar depresión, en tanto que un mayor miedo a revelar emociones propias contribuye a mayor sintomatología depresiva.

En el modelo creado para predecir la somatización (Tabla 4) se evidencia que entre las variables controladas, solo el sexo hace una contribución significativa, revelando que las mujeres presentan más sintomatología somática. El miedo a la revelación nuevamente predice, en forma significativa, la somatización, por lo que a mayor miedo a revelar las propias emociones, experiencias y sentimientos, mayor la sintomatología física por parte de los desempleados. El término de interacción entre el apoyo social percibido y la duración del desempleo resultó significativo, lo cual indica que este tipo de apoyo social modera la relación entre el estrés del desempleo (mayor duración) y la aparición de síntomas físicos.

En este caso, las personas que perciben un alto apoyo social (más 1 DT de la media) reportan menos sintomatología física, cuando el tiempo desempleados aumenta $(b=0.17, t=1.94, p=$ 0.05). Por su parte, las personas con un bajo apoyo social percibido (menos 1 DT de la media) presentan menor índice de somatización que el resto de los participantes, cuando tienen poco tiempo desempleados, pero mientras más tiempo llevan en esa condición hay un mayor deterioro en su sintomatología física $(b=-0.21, t=-1.83, p=0.068)$, aunque esta diferencia haya sido solo marginal. 
TABLA 3

Modelo de regresión jerárquica para la predicción de la Depresión

\begin{tabular}{|c|c|c|c|c|c|}
\hline & Modelo & Coeficiente no Estandarizado & $R^{2}$ corregida & Cambio en $R^{2}$ & Cambio en $F$ \\
\hline \multirow[t]{3}{*}{1} & Paso 1 & & 0.002 & 0.002 & 0.343 \\
\hline & Sexo & -0.070 & & & \\
\hline & Edad & -0.004 & & & \\
\hline \multirow[t]{2}{*}{2} & Paso 2 & & 0.007 & 0.004 & 1.198 \\
\hline & Duración del desempleo & -0.073 & & & \\
\hline \multirow[t]{4}{*}{3} & Paso 3 & & 0.148 & 0.162 & $18.416 * *$ \\
\hline & Afrontamiento Social & $0.110 *$ & & & \\
\hline & Miedo a la Revelación & $-0.369 * *$ & & & \\
\hline & Apoyo Social Percibido & 0.044 & & & \\
\hline \multirow[t]{4}{*}{4} & Paso 4 & & 0.143 & 0.004 & 0.424 \\
\hline & Afrontamiento Social x DD & -0.051 & & & \\
\hline & Miedo a la Revelación x DD & 0.037 & & & \\
\hline & Apoyo Social Percibido x DD & 0.075 & & & \\
\hline
\end{tabular}

$\mathrm{DD}=$ Duración del Desempleo $* * p<0.01 ; p<0.05$

Fuente: elaboración propia.

\section{Figura 1}

Interacción entre apoyo social percibido y duración del desempleo en su efecto sobre la salud general

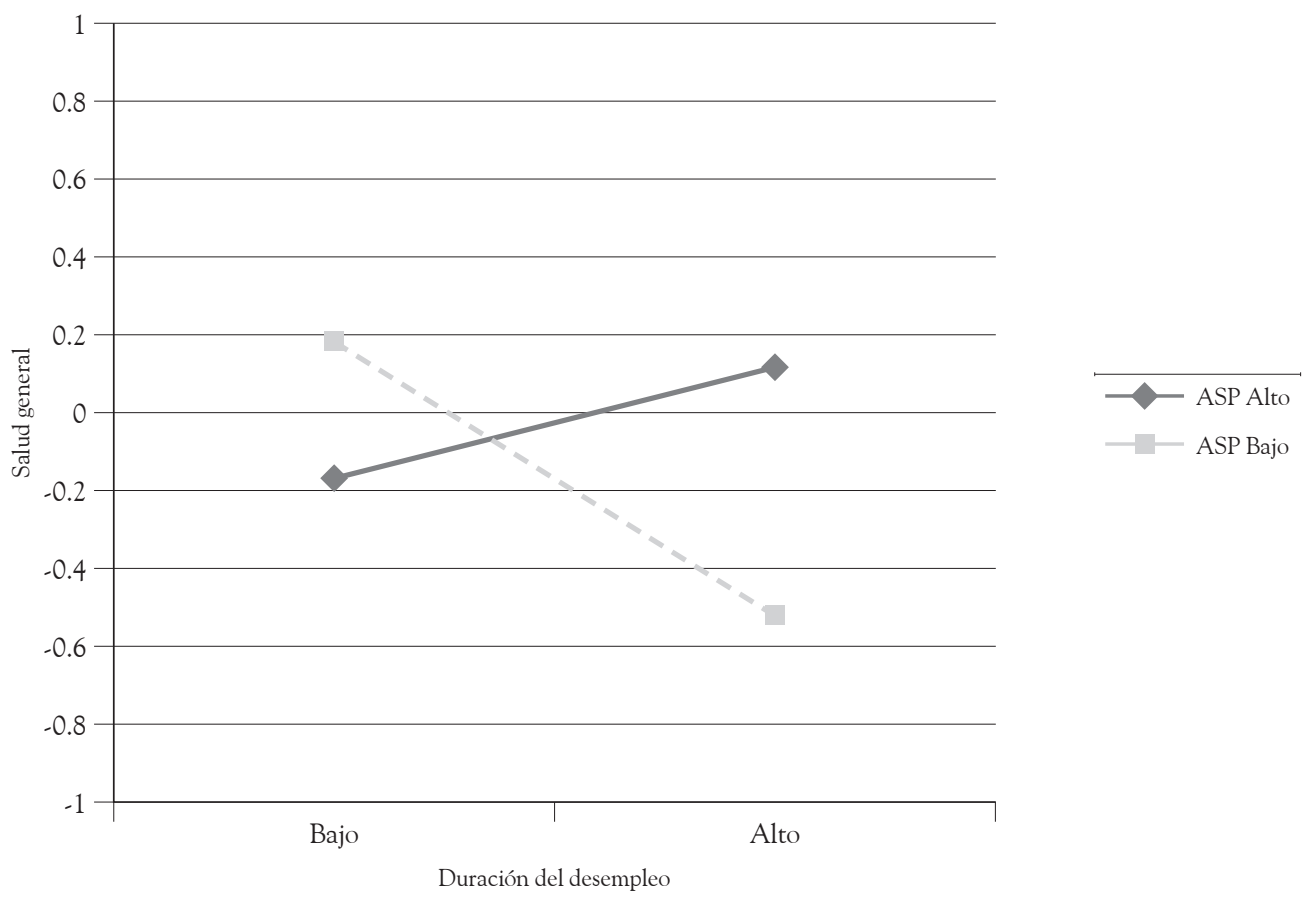

Fuente: www.namaocerta.org.br

\begin{tabular}{|l|l|l|l|l|} 
| Universitas Psychologica & V. 10 & No. 3 & Septiembre-diciembre & 2011
\end{tabular} 
TABLA 4

Modelo de regresión jerárquica para la predicción de la Somatización

\begin{tabular}{|c|c|c|c|c|c|}
\hline & Modelo & Coeficiente no Estandarizado & $R^{2}$ corregida & Cambio en $R^{2}$ & Cambio en $F$ \\
\hline \multirow[t]{3}{*}{1} & Paso 1 & & 0.006 & 0.016 & 2.365 \\
\hline & Sexo & $0.170 *$ & & & \\
\hline & Edad & -0.058 & & & \\
\hline \multirow[t]{2}{*}{2} & Paso 2 & & 0.005 & 0.002 & 0.682 \\
\hline & Duración del desempleo & -0.053 & & & \\
\hline \multirow[t]{4}{*}{3} & Paso 3 & & 0.037 & 0.042 & $4.283^{* *}$ \\
\hline & Afrontamiento Social & 0.073 & & & \\
\hline & Miedo a la Revelación & $-0.185 * *$ & & & \\
\hline & Apoyo Social Percibido & 0.006 & & & \\
\hline \multirow[t]{4}{*}{4} & Paso 4 & & 0.077 & 0.048 & $5.074 * *$ \\
\hline & Afrontamiento Social x DD & 0.038 & & & \\
\hline & Miedo a la Revelación x DD & -0.045 & & & \\
\hline & Apoyo Social Percibido x DD & $0.191 * *$ & & & \\
\hline
\end{tabular}

$\mathrm{DD}=$ Duración del Desempleo

$* * p<0.01 ; * p<0.05$

Fuente: elaboración propia.

FIGURA 2

Interacción entre apoyo social percibido y duración del desempleo en su efecto sobre la somatización

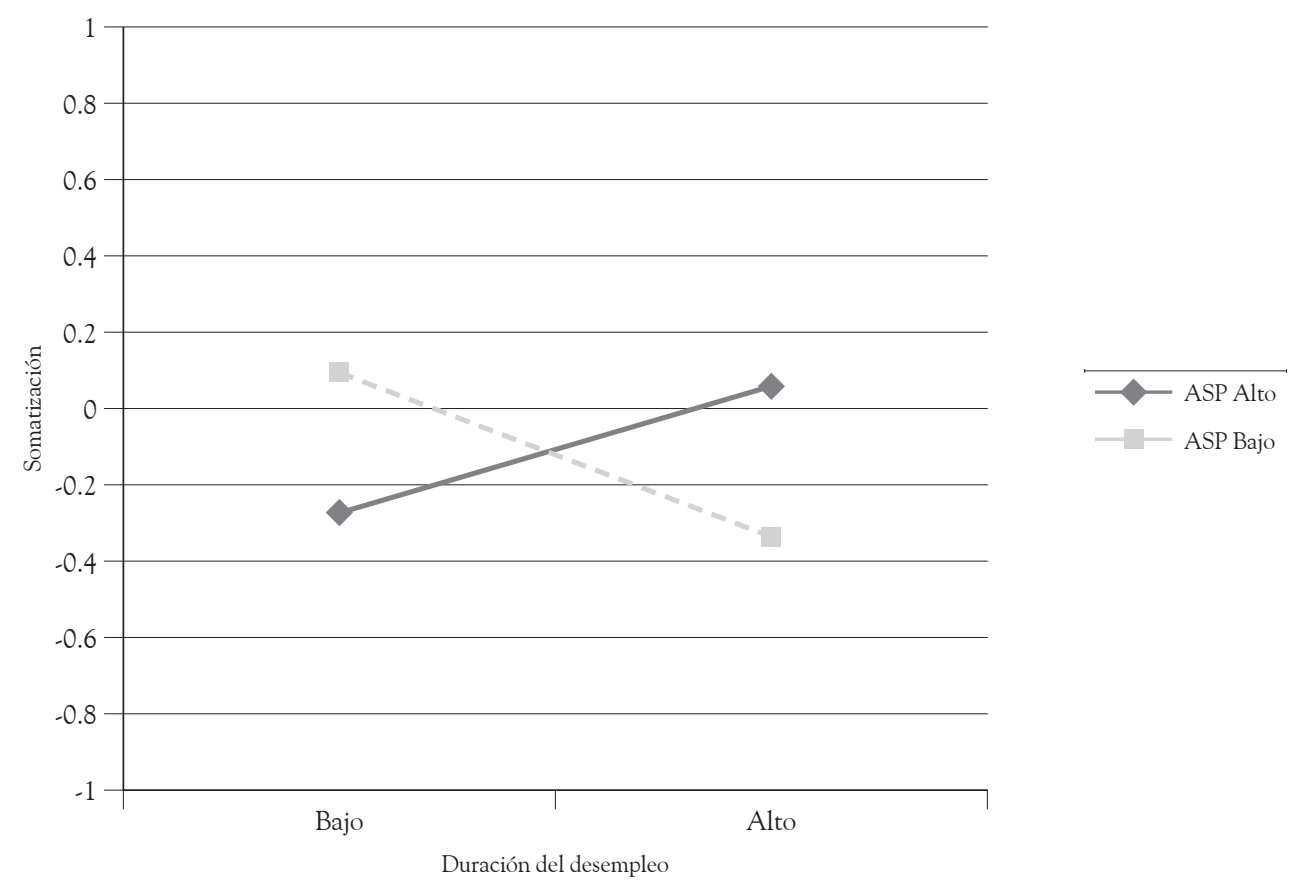

Fuente: www.namaocerta.org.br 
En la predicción de la ansiedad (Tabla 5) se evidencia que a mayor miedo a revelar las propias emociones, mayor presencia de síntomas de ansiedad en los desempleados. Sin embargo, los resultados de esta ecuación indicaron que no es posible verificar el efecto de moderación del apoyo social en la relación entre la duración del desempleo y la aparición de síntomas de ansiedad.

En la predicción de la disfunción social, modelo resumido en la Tabla 6 , se observa que las personas de mayor edad reportan más desajuste social, siendo esta la única relación que resultó significativa en la predicción de la disfunción social, sin encontrar evidencias en apoyo a la hipótesis de moderación del apoyo social, en sus tres dimensiones, con respecto a este indicador de salud.

\section{Discusión}

El objetivo de este estudio ha sido el de evaluar el posible rol moderador del apoyo social, tanto dis- posicional como percibido, en la relación entre el estrés del desempleado y su salud. En este trabajo se ha asumido el tiempo o duración del desempleo como el evento o estímulo estresor que formaría parte del modelo de moderación.

Los resultados indicaron que los participantes que conformaron la presente muestra de desempleados, cuentan con redes de apoyo moderadamente fuertes, así como manifiestan usar el apoyo social cuando las circunstancias lo requieren, aunque la comparación por géneros mostró que son las mujeres quienes perciben significativamente más apoyo del entorno que los hombres. Esto, sin embargo, no parece traducirse en un mayor bienestar para las mujeres, puesto que los hombres desempleados mostraron un menor deterioro físico y psicológico que las mujeres en esta condición. Estos resultados contradicen el conocimiento común según el cual el desempleo es más dañino para la salud mental de los hombres.

TABLA 5

Modelo de regresión jerárquica para la predicción de la Ansiedad

\begin{tabular}{|c|c|c|c|c|c|}
\hline & Modelo & Coeficiente no Estandarizado & $R^{2}$ corregida & Cambio en $R^{2}$ & Cambio en $F$ \\
\hline \multirow[t]{3}{*}{1} & Paso 1 & & 0.009 & 0.019 & 2.793 \\
\hline & Sexo & 0.160 & & & \\
\hline & Edad & -0.092 & & & \\
\hline \multirow[t]{2}{*}{2} & Paso 2 & & 0.006 & 0.001 & 0.197 \\
\hline & Duración del desempleo & -0.029 & & & \\
\hline \multirow[t]{4}{*}{3} & Paso 3 & & 0.057 & 0.060 & $6.241 * *$ \\
\hline & Afrontamiento Social & 0.016 & & & \\
\hline & Miedo a la Revelación & $-0.231^{* *}$ & & & \\
\hline & Apoyo Social Percibido & 0.037 & & & \\
\hline \multirow[t]{4}{*}{4} & Paso 4 & & 0.056 & 0.008 & 0.840 \\
\hline & Afrontamiento Social x DD & -0.031 & & & \\
\hline & Miedo a la Revelación x DD & -0.011 & & & \\
\hline & Apoyo Social Percibido x DD & 0.113 & & & \\
\hline
\end{tabular}

$\mathrm{DD}=$ Duración del Desempleo

$* * p<0.01 ; * p<0.05$

Fuente: elaboración propia. 
TABLA 6

Modelo de regresión jerárquica para la predicción de la Disfunción Social

\begin{tabular}{|c|c|c|c|c|c|}
\hline & Modelo & Coeficiente no Estandarizado & $\mathrm{R}^{2}$ corregida & Cambio en $\mathrm{R}^{2}$ & Cambio en $\mathrm{F}$ \\
\hline \multirow[t]{3}{*}{1} & Paso 1 & & 0.043 & 0.052 & $7.959 * *$ \\
\hline & Sexo & 0.159 & & & \\
\hline & Edad & $-0.214 * *$ & & & \\
\hline \multirow[t]{2}{*}{2} & Paso 2 & & 0.050 & 0.011 & 3.389 \\
\hline & Duración del desempleo & -0.119 & & & \\
\hline \multirow[t]{4}{*}{3} & Paso 3 & & 0.058 & 0.017 & 1.772 \\
\hline & Afrontamiento Social & 0.106 & & & \\
\hline & Miedo a la Revelación & -0.035 & & & \\
\hline & Apoyo Social Percibido & 0.044 & & & \\
\hline \multirow[t]{4}{*}{4} & Paso 4 & & 0.053 & 0.005 & 0.506 \\
\hline & Afrontamiento Social x DD & -0.088 & & & \\
\hline & Miedo a la Revelación x DD & -0.040 & & & \\
\hline & Apoyo Social Percibido x DD & 0.051 & & & \\
\hline
\end{tabular}

$\mathrm{DD}=$ Duración del Desempleo

$* * p<0.01 ; * p<0.05$

Fuente: elaboración propia.

En este sentido, McKee-Ryan, Song, Wanberg y Kinicki (2005) tienen dos hipótesis alternativas para explicar estos resultados. Una de ellas es que la diferencia entre géneros solamente esté mostrando los hallazgos generales según los cuales las mujeres reportan peor salud mental que los hombres, independientemente del contexto en el sean evaluadas. La segunda explicación, un poco más compleja, se basa en investigaciones recientes que sugieren que los cambios en los roles de género han permitido que el trabajo tenga un papel más central en la vida e identidad de las mujeres trabajadoras (Lee \& Owens, 2002; Waters \& Moore, 2002), generando la pérdida del mismo mayor distrés en estas con su consecuente deterioro, sobre todo si en este grupo el tiempo de desempleo es significativamente mayor. Sin embargo, es necesario realizar más investigaciones para indagar los factores que contribuyen al deterioro diferencial en la salud de los hombres y las mujeres desempleados, conside- rando por ejemplo la identificación con el trabajo como un factor moderador (Brown, 1996).

Fue también interesante observar cómo los desempleados de mayor edad significativamente perciben tener menor apoyo de su contexto, indicando esto, de alguna manera, el debilitamiento de las redes de soporte social a medida que se avanza en edad y pudiendo esta condición determinar el mayor deterioro observado en este grupo en cuanto a su funcionamiento social y salud general. En congruencia con estos resultados, Kulik (2001) encontró que las personas entre los 36 y 52 años tuvieron un mayor deterioro en su salud percibida, que los más jóvenes.

Muy probablemente, la explicación a dicho fenómeno se encuentre en el hecho de que las personas de edad media (35 a 50 años) se encuentran en el periodo más productivo de su vida, están en un momento en el que deben mantener el hogar, criar hijos y ser útiles para la sociedad, en comparación 
a los más jóvenes que usualmente no tienen dichas responsabilidades, ni las presiones sociales para adquirirlas. Así, es muy probable que el funcionamiento social, entendido como la medida en la que la persona se ha podido mantener activa, tomando decisiones importantes y preservando las relaciones de su vida cotidiana, se vea más afectado en personas de mayor edad que están desempleadas, con el agravante de percibir que el contexto está menos disponible para brindarles soporte.

Los resultados con respecto a la relación entre la duración del desempleo y el miedo a la revelación (dimensión del ITQ) van en el mismo sentido, en función de que los desempleados que más tiempo han permanecido en esta condición, manifiestan mayor temor y dificultad para expresar sus emociones y sentimientos, mostrando correlativamente peor funcionamiento social y mayor deterioro general. Son estos resultados con respecto a las relaciones entre la salud y el apoyo social, los que precisamente justificaron la exploración sobre el posible rol moderador del apoyo social, tanto en su concepción individual como contextual.

En este sentido, los análisis de regresión permitieron determinar que el apoyo social percibido funcionó como moderador de la relación entre la duración del desempleo y la salud, tanto para su puntaje general -bienestar general-como para el índice de somatización (los resultados no fueron significativos para la ansiedad o la depresión). Esto indica que los desempleados que pasan más tiempo en esta condición y al mismo tiempo perciben menos soporte social de parte de su contexto, manifiestan mayor deterioro en su salud física y psicológica general y somatizan más que aquellos con apoyo social percibido más alto. Hallazgos previos en muestras de desempleados habían ya establecido el papel amortiguador del apoyo social percibido, sobre todo en su impacto sobre la somatización (p. ej., Gore, 1978; Gascón et al., 2003; Howe et al., 2004), aunque no se contaba con evidencias sobre estas relaciones en muestras de desempleados latinoamericanos.

Las evidencias sobre el rol moderador del apoyo social en la relación estrés-salud en diferentes grupos de individuos, especialmente la acción protec- tora del apoyo social percibido son numerosas ( $\mathrm{p}$. ej., Chan, 2002; Cobb, 1976; Cohen \& Hoberman, 1983; Cohen \& Wills, 1985; Sarason, 1981; Schmidt \& Andrykowsi, 2004). Específicamente, Gottlieb (1985) encontró que los individuos que tienen un alto apoyo social percibido, generalmente evalúan su entorno como menos amenazante, lo cual les facilita implementar formas de afrontamiento menos emocionales, a la vez que más activas y racionales. Precisamente, este razonamiento describe el modelo de "amortiguación" del apoyo social, según el cual este actúa protegiendo al individuo durante los momentos de estrés, potenciando la adaptación (por ejemplo, mediante la facilitación o mejora de las conductas de afrontamiento). En este sentido, el apoyo social percibido puede reducir el impacto del estrés: 1) eliminando o reduciendo el propio estresor, 2) reforzando la capacidad del individuo para hacer frente al estresor, 3) atenuando la respuesta del estrés una vez este se ha puesto en marcha (Sandín, 1995; Taylor \& Aspinwall, 1996). En el caso de la muestra de desempleados bajo estudio, fue precisamente el poco apoyo percibido por parte de estos en la circunstancia de vivir largos períodos de desempleo, lo que desencadenó un deterioro en su salud general, a la vez que una percepción de fuerte apoyo en ese mismo contexto, redujo significativamente los índices de somatización con respecto a sus contrapartes.

Lo destacado del presente estudio ha sido determinar las diferencias en el efecto moderador que diversas dimensiones del apoyo social pueden tener sobre la salud de los desempleados, sobre todo al comparar dimensiones de apoyo social disposicional, entendido como la tendencia de los individuos a buscar apoyo, con respecto al soporte que perciben por parte de su entorno. En este sentido, las subdimensiones del apoyo social disposicional, miedo a la revelación y afrontamiento social, respectivamente, en este estudio no mostraron que moderan la relación entre el estrés del desempleo y el estatus de salud de los participantes, habiendo sido solo el indicador de apoyo social percibido el que mostró tales efectos.

Tal como se indicó anteriormente, las presentes evidencias sugieren que el factor que ejerce más 
efecto amortiguador es, precisamente, el percibir que se cuenta con apoyo, más que la habilidad y los propios recursos para buscar ese apoyo cuando se requiere, coincidiendo con lo expresado en estudios previos sobre el poder del apoyo percibido, por encima de otras formas de apoyo (p. ej., material, informativo, emocional, etc.) (Barrera, 1981; Cohen \& Hoberman, 1983; Procidano \& Heller, 1983). Una evidencia de la poca relación entre el apoyo disposicional y el apoyo percibido para los participantes de este estudio, fueron las bajas correlaciones obtenidas entre sus dimensiones (para Afrontamiento Social $r_{\mathrm{xy}}=0.21, p<0.01$ y para Miedo a la Revelación $r_{\mathrm{xy}}=-0.30, p<0.01$ ). Son en esencia las condiciones del entorno de estos desempleados las que ejercen mayor impacto sobre su salud, bajo largos períodos de desempleo, en comparación con sus características individuales, las cuales, como el miedo a la revelación, parecieran tener un efecto negativo directo sobre la salud sin importar la condición en que se encuentre el desempleado. No se encontraron evidencias previas de estudios que hubieran hecho una comparación similar entre formas de apoyo social en su efecto de amortiguación, por lo cual se sugiere seguir investigando la naturaleza de esta diferencia con respecto a los efectos de moderación en la relación estrés-salud, no solo en desempleados sino en otras poblaciones.

\section{Referencias}

Aiken, L. \& Weist, S. (1991). Multiple regression: Testing and interpreting interactions. Newbury Park, CA: Sage.

Álvaro, J. (1992). Desempleo y bienestar psicológico. Madrid: Siglo XXI.

Artazcoz, L., Benach, J., Borrell, C. \& Cortès, I (2004). Unemployment and mental health: Understanding the interactions among gender, family roles, and social class. American Journal of Public Health, 94 (1), 82-88.

Baron, R. M. \& Kenny, D. A. (1986). The moderator mediator variable distinction in social psychological research-conceptual, strategic, and statistical considerations. Journal of Personality and Social Psychology, 51, 1173-1182.
Barrera, M. (1981). Social support in the adjustment of pregnant adolescents: Assessment issues. En B. H. Gottlieb (Ed.), Social network and social support (pp. 69-96). Beverly Hills, CA: Sage.

Brown, S. (1996). A meta-analysis and review of organizational research on job involvement. Psychological Bulletin, 120 (2), 235-255.

Chan, D. (2002). Stress, self-efficacy, social support, and psychological distress among prospective Chinese teachers in Hong Kong. Educational Psychology, 22 (5), 557-569.

Cobb, S. (1976). Social support as moderator of life stress. Psychosomatic Medicine, 38, 300-314.

Cohen, S. \& Wills, T. (1985). Stress, social support and the buffering hypothesis. Psychological Bulletin, 98, 310-357.

Cohen, S. \& Edwards, J. R. (1989). Personality characteristics as moderators of the relationship between stress and disorder. En R. W. J. Neufeld (Ed.), Advances in the investigation of psychological stress (pp. 235-283). New York: John Wiley \& Sons, Inc.

Cohen, S. \& Hoberman, G. M. (1983). Positive events and social support as buffers of life change stress. Journal of Applied Social Psychology, 13, 99-125.

Dooley, D., Fielding, J. \& Lennart, L. (1996). Health and unemployment. Annual Review of Public Health, 17, 449-465.

Forbes, A. \& Roger, D. (1999). Stress, social support and fear of disclosure. British Journal of Health Psychology, 4, 165-179.

Gascón, S., Olmedo, M., Bermúdez, J., García-Campayo, J. \& Ciccotelli, H. (2003). Estrés por desempleo y salud. Cuadernos de Medicina Psicosomática y Medicina de Enlace, 66, 9-18.

Gore, S. (1978). The effect of social support in moderating the health consequences of unemployment. Journal of Health and Social Behavior, 19, 157-165.

Gottlieb, B. (1985). Social support and the study of personal relationship. Journal of Social and Personal Relationship, 2, 351-375.

Guarino, L. (2004). Emotional Sensitivity: a new measure of emotional lability and its moderating role in the stress-illness relationship. Tesis Doctoral sin publicar, Universidad de York, Inglaterra.

Guarino, L. \& Sojo, V. (2009). Adaptación y validación del ITQ (Interpersonal Trust Questionnaire). Una 
nueva medida del Apoyo Social. Avances en Psicología Latinoamericana, 27, 192-206.

Howe, G., Lockshin, M. \& Caplan, R. (2004). Job loss and depressive symptoms in couples: Common stressors, stress transmission, or relationship disruption? Journal of Family Psychology, 18 (4), 639-650.

Kulik, L. (2001). Impact of length of unemployment and age on jobless men and women: A comparative analysis. Journal of Employment Counseling, 38 (3), 15-27.

Lee, C. \& Owens, R. G. (2002). Men, work, and gender. Australian Psychologist, 37, 13-19.

McKee-Ryan, F., Song, Z., Wanberg, C. \& Kinicki, A. (2005). Psychological and physical well-being during unemployment: A meta-analytic study. Journal of Applied Psychology, 90 (1), 53-76.

Molina, J. \& Andrade, C. (2002). La estructura factorial del GHQ-60 en una muestra de población general: una versión escalar para población española. Revista Internacional On-line, 1 (2). Recuperado el 20 de abril de 2005, de http://www.bibliopsiquis. com/asmr/0102/0102lef.htm

Preacher, K. J., Curran, P. J. \& Bauer, D. J. (2006). Computational tools for probing interaction effects in multiple linear regression, multilevel modeling, and latent curve analysis. Journal of Educational and Behavioral Statistics, 31, 437-448.

Procidano, M. \& Heller, K. (1983). Measures of perceived social support from friends and from family: Three validation studies. American Journal of Community Psychology, 11, 1-24.

Rincón, R. (2003). Estrategias de afrontamiento ante el desempleo: análisis de un conjunto de fuentes y su impacto en la salud mental. Tesis de pregrado no publicada, Universidad Central de Venezuela, Caracas.

Sandín, B. (1995). El estrés. En A. Bellock, B. Sandín \& F. Ramos (Eds.), Manual de Psicopatología (Vol. 3, pp. 3-52). Madrid: McGraw Hill.

Sarason, I. (1981). Test anxiety, stress and social support. Journal of Personality, 49, 101-114.

Schmidt, J. \& Andrykowski, M. (2004). The role of social and dispositional variables associated with emotional processing in adjustment to breast cancer an internet-based study. Health Psychology, 23 (3), 259-266.

Steptoe, A. (1991). Psychological coping, individual differences and physiological stress responses. En C. L. Cooper \& R. Payne (Eds.), Personality and Stress: Individual differences in the stress process (pp. 205-233). New York: John Wiley \& Sons, Inc.

Taylor, S. \& Aspinwall, L. (1996). Mediating and moderating processes in psychosocial stress. En $\mathrm{H}$. Kaplan (Ed.), Psychosocial Stress. Perspectives on structure, theory, life-course and methods (pp. 71110). San Diego, CA: Academic Press.

Ullah, P., Banks, M. \& Warr, P. (1985). Social support, social pressures and psychological distress during unemployment. Psychological Medicine, 15, 283. 295.

Waters, L. \& Moore, K. (2002). Predicting self-esteem during unemployment: The effect of gender, financial deprivation, alternate roles, and social support. Journal of Employment Counseling, 39 (12), 171-189. 
